\title{
Identification in Drug Prescription using Artificial Intelligence
}

\author{
*Alekhya Roy \\ Dept. of Information Technology, MPSTME NMIMS (DEEMED TO BE UNIVERSITY) Maharashtra,India
}

\begin{abstract}
Medication errors are one of the safety problems most frequently seen in hospital organizations. It is estimated that $12.2 \%$ of all hospitalized patients are involved in some form of adverse drug event (ADE). A significant amount of ADEs result from handing the incorrect drug to a patient or prescribing the wrong medication. Issuing of correct prescriptions is a foundation of patient safety. Many times it has happened thatdue to the doctor's illegible handwriting, wrong prescriptions are bought by the customer. In this paper, variousmethods using artificial intelligence to identify drugs prescribed for patients and to minimise errors which havebeen happening before.
\end{abstract}

Keywords: Look-Alike, Sound-Alike, Optical Identification, Pill Localisation, You Only Look Once

\section{Introduction}

Giving of right medications is a reinforcement of patient safety. Medication mistakes address one of the main issues in medical services, with 'look-alike and sound-alike' (LASA) being the lead error. There is also prescription errors which are ordinarily occasions that get from slips, passes, or errors, for instance, writing a dosage that is significant degrees higher or lower than the right one due to mistaken computation, or wrong solution because of similitudes in drug brand names or drug names. Commonly it has happened that because of the specialist's illegible handwriting, wrong prescriptions are purchased by the customer.

Deep learning methods have revolutionised recognizable identification classifiers in many fields. A significant part of the AI work in medical care is engaged around illness expectation in clinical settings, which is a significant application that still can't seem to convey vigorously. Notwithstanding, there are other basic viewpoints like aiding patients and mind groups associate and convey in proficient and significant ways, which could convey fourfold point improvements. The biggest subset of clinical blunders is medicine mistake. Giving the right treatment plan to patients incorporates information about their present prescriptions and medication sensitivities, a regularly difficult assignment. The boundless development of endorsing and consuming prescriptions has expanded the requirement for applications that help medicine reconciliation. Thusly, a more prompt effect of AI in medical services will happen with a consistent incorporation of AI into clinical work processes, promptly tending to healthcare.

Various solutions have been proposed, similar to AI Optical identification and graphic recognition technology, how CNN can be applied for drug identification and how this work fills in as an illustration that one of the underlying effects of AI in medical care will be to smooth out assignments, prior to getting into replacing care staff in complicated and high-stake errands, like diagnosing and prognosticating, if at any time. It additionally discusses pill recognition neural network systems like ResNet50, MobileNet, Inception V3[1]. There is likewise the arrangement of You Only Look Once (YOLO), a deep learning structure which is, although a more extended way, yet all at once less expensive and more dependable. This fundamentally can lessen the tremendous mistakes of Look Alike-Sound Alike (LASA).

Section 2 describes the current problems in drug identification using artificial intelligence.

Section 3 describes the possible solutions for the problems and issues faced in section 2, while section 4 will comprise of the features and their solutions, which will be compared. 


\subsection{Mistakes made by pharamicists in recording drug prescription information of patient.}

Generally, the patient gets their own medicine, they should bring their own medication prescription andrequest that the drug specialist to adjust. The drug specialist has the obligation to assess the medication, the association status, and the record of speaking with the specialist. In any case, in the execution, whilethe drug specialist performs wellbeing drug store care, it isn't difficult to record the medication solution data of the patient, essentially on the grounds that the drug store data content of the drug store care is extremely complicated. To give proper medical services and stay away from prescription blunders, it is important to know which drugs a patient is taking. It is regularly quite difficult for consumers to distinguish pills when pills are moved to various compartments, consolidated to a solitary holder for comfort, or divided into day-of-week pillboxes to work taking drugs the board. While for the most part all around expected, when patients separate drugs from their unique containers or bundling, this presentsa test to their medical care groups. The unseen side-effect today is that the patient might encounter wideranges in pill appearance when topping off a drug because of changes in nonexclusive brands. This prompts considerably more disarray and complicacy when a medication is recommended, which might prompt slip-ups made by the drug specialists.

\subsection{Mistakes made due to the difficulty in handling increasing number of medicine consumers.}

The greatest number of clinical mistakes according to a study from the Institute of Medicine, which observed that the most widely recognized kind of preventable clinical blunder is a prescription mistake, which results in over 1.5-m wounds and more than \$3b in complexity costs alone. The Institute of Medicine's 2006 report further gives rules on lessening the high recurrence and unsatisfactory expenseof prescription blunder, including more prominent utilization of data innovation, which could be carried out at each stage from recommending and administering through to checking the patient's reaction. Innovation arrangements have been applied to medicate reference data, drug-drug collaborations, drug sensitivities, and limit admonitions for high dosages. Regardless of the normal event, there is little examination subsidizing in clinical blunder, especially when contrasted and other driving reasons for death, like coronary illness and disease. In any case, there are clear money saving advantages; automated drug frameworks can possibly decrease mistakes by $84 \%$ and save medical clinics more than $\$ 500 \mathrm{k} /$ year in direct costs.

Forward leaps in doctor prescribed medicine are among the reasons we live longer. Simultaneously, physician-recommended medicine use is expanding; Medicine use is expanding across age gatherings, especially among the older. Of individuals who matured 65 or more, nine of ten were on doctor- prescribed drugs inside the most recent 30 days. In a similar age bunch, the utilization of various drugsis normal.

\subsection{The "Look-Alike, Sound-Alike" error.}

Out of the relative mistakes made by people in giving some unacceptable medication to the patient, the Look-Alike, SoundAlike error is the most well-known one. The presence of confusing medication names is one of the most well-known reasons for prescription mistake and is of concern around the world. Witha huge number of medications as of now available, the potential for mistake because of so many medication names are critical. Adding to this disarray are unintelligible handwriting, inadequate information on drug names. For instance, the medications Losec (omeprazole) and Lasix (furosemide) are tricky around the world. Moreover, world administrative specialists and the worldwide drug industryshould put more accentuation on the security issues related with drug names. The expanding potential for LASA drug errors was featured in the Joint Commission's Sentinel Event Alert in the United States of America and was fused into the Joint Commission's National Patient Safety Goals. Different proposals pointed toward limiting name errors are opinionated; genuinely isolating meds with LASA names in all capacity regions; including both the brand name and non-restrictive name taking drugs requests to give overt repetitiveness; and utilizing "tall man" (mixed case) lettering (for example Dopamine versus Dobutamine) to underline drug name contrasts. By joining measures, for example, these, medical services 
associations can extraordinarily lessen the danger for LASA medicine blunders. While numerous LASA blunders happen in medical clinics, the issue is essentially as extraordinary in short term care settings, which require a similar level of thoroughness in carrying out hazard decrease procedures.

\section{Solutions and countermeasures}

\subsection{Solution for mistakes by pharmacists in keeping medicinal records}

Artificial Intelligence techniques, like AI Optical Identification, can be utilized in helping drug specialists to perform drug store administrations, can distinguish the patient's clinical record data through solution and recognizable proof, and utilize the man-made consciousness optical ID to gather the clinical records. Optical Identification study gathered the solutions of 294 clinical foundations, and through the distinguishing proof of fake medication data, the implementation of neural network artificial intelligenceidentification learning, can achieve more than $98 \%$ of drug information area identification results., The artificial intelligence technical assistance system can judge the drug information area in each prescription, and then use optical identification technology to interpret the drug information. It can likewise build up a medication data information base to aid the correlation of optical ID results. The consequences of this study can be partitioned into three sections, the initial segment, the aftereffects of prescription collection, the neural network learning to determine the drug information location, the thirdpart of the text optical identification and matrix listing drug information.

\subsection{Solution for managing increasing number of medicine consumers}

For the implementation of pill recognition service, a Resnet50 model was used as the final classifier. Both MobileNet and SqueezeNet are simpler deep CNN-based classifiers aimed at running in mobile devices, which provide an interesting contrast with the initial ResNet50 selection. On the other hand, InceptionV3 has a network structure of comparable depth and number of parameters. The results of thisevaluation are the basis from which will select the type of model to power the next iteration of the recognition service. Current results indicate that ResNet50 continues to be a good option, with InceptionV3 and surprisingly MobileNet also having comparable performance in this domain.

The method used to perform could be:

\section{Data and preprocessing}

The images must be divided into reference images and consumer-quality ones. Reference images have controlled lighting and background, while the consumer-quality ones have variable conditions. Consumer images additionally vary in focus and device type. They imitate the images taken by users thatwould be sent to an automated pill-recognition system. 


\section{Identification system overview}

Pills are identified employing two deep-learning models in series. First, perform image segmentation isolating the pill from the background with a blob-detection CNN technique and define a bounding box to crop a smaller image that centres on the pill. After that, use a deep-learning-based classifier to return a ranked list of drug codes based on matched likelihood by the pill in the cropped image output by the initial stage.

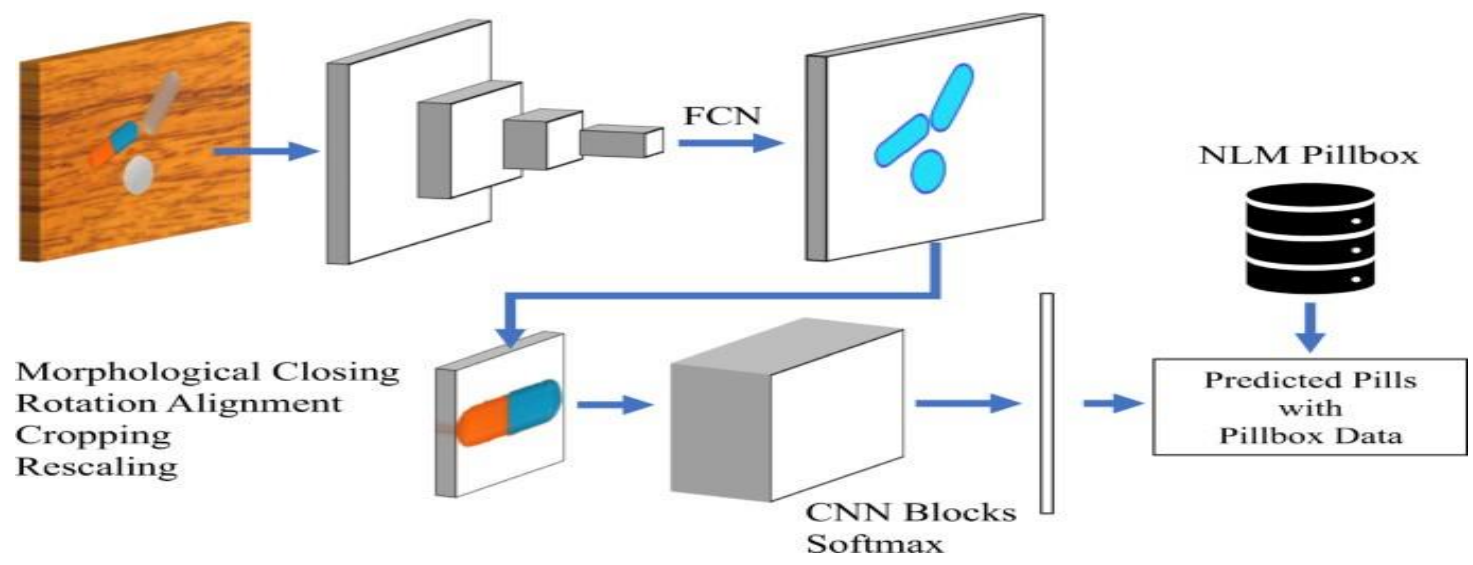

An overview of pill detection and classification approach.

\section{Pill localization}

The pill localization approach is basically a blob-detection neural network and morphological post processing. For the blob detector, a fully convolutional network $(\mathrm{FCN})$, which is a pixel-segmentation algorithm, was trained.

\section{Synthetic training images}

Synthetic images of medicines to be generated. The pill images can be superimposed on the background images with the following parameters:

- $\quad 1-5$ pills per image

- $360^{\circ}$ rotation

- $\quad 20-50$ px pill size

- $\quad$-20 px drop shadow

During the model training runtime, various image augmentations were applied, including contrast and brightness adjustment, Gaussian-blur, and affine and perspective transformation to increase variety in the training set.

\section{CNN models for identification}

Deep CNNs have been proven successful in multiple tasks identifying object categories in pictures taken with realistic conditions of varying illumination, focus, and perspective. The main goal of this work is toreliably identify pills from images under any imaging condition to provide accurate medication information. 


\section{Pill-identification service implementation}

The implementation of the pill identification service is composed of two web-based APIs that handle segmentation first and then identification. The APIs are hosted in separate Azure VMs[3] with a Python implementation based on the Flask framework, and using models with a TensorFlow back end. The segmentation service locates pills in the image it receives as a parameter. It responds with a list of bounding-box and confidence-score pairs for all the pills it has found. The client is then responsible for cropping the source image based on the received bounding boxes to obtain pill-centred images. Requeststo the identification service also require an image as a parameter. These are expected to be generated from the first API, since the identification model is trained and validated on images generated with it. Using this platform, drug identification will become much faster and accurate, without human errors as much as possible.

\subsection{Solution for the "Look-Alike, Sound-Alike" error}

With the speed of progress in deep learning techniques, it is expected that more accurate deep learning solutions and frameworks will emerge to distinguish subtle image features among different object types, thus reducing if not solving the major error caused by look-alike sound-alike. A novel deep learning drug identification (DLDI) model that delivered satisfactory results for drug identification based on images of blister packages. The CNN simulates the response of neurons in the human brain to signals byperforming various mathematical operations on features to complete the classification. Repetition of these processes achieves the purpose of recognition/

\section{Software and hardware devices}

This study used You Only Look Once (the abbreviation 'YOLO' having been proposed by Redmon et al. in 2015) as the solution framework for deep learning. YOLO focuses on both the area prediction partof detection and the category prediction part for classification. YOLO integrates detection and classification into the same neural network model, with fast and accurate target detection and recognition.

\section{Outcome measurement}

Image based techniques, being non-intrusive and without resort to additional devices like RFID tag or bar code, have been a preferred solution to object identification problems. Conventional image-based solutions by computer vision rely on welldefined hierarchical features for effective comparison.

The CNN simulates the response of neurons in the human brain to signals by performing various mathematical operations on features to complete the classification. Repetition of these processes achieves the purpose of recognition. Deep learning allows learning of features automatically, without the need to define features of drugs before machine learning. This advantage eliminates human error and assists pharmacists to identify medicines correctly.

The goal was to illustrate how 'look-alike' error can be captured and explained by a convolution-based deep learning network whose working mechanism is much similar to the human visionary recognition capability. Subsequently, appropriate solution to extract more detailed subtle differences can be utilizedin differentiating between look-alike objects.

\section{Analysis and ReviewSolutions:}

\section{- AI Optical identification (OCR):}

Combining $\mathrm{AI}$ and OCR together is proving to be a winning strategy for both data capture and management

Today, OCR platforms are still used to convert handwritten or printed text into machine-encoded text sothat it can be accessed on a computer 


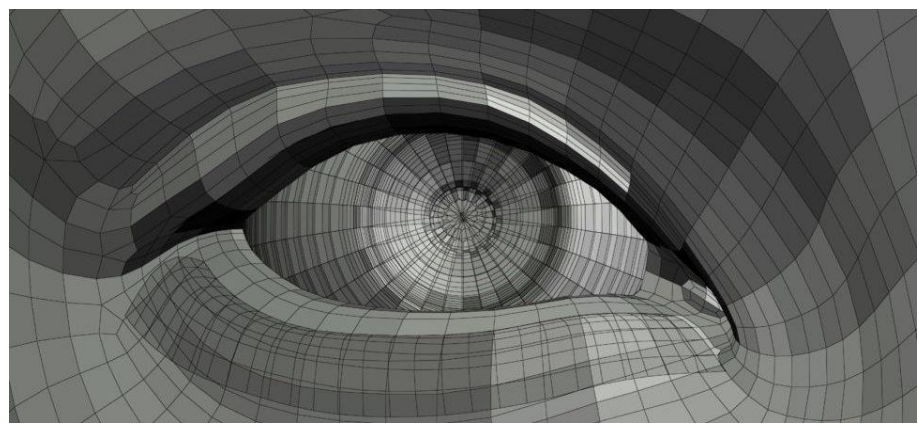

The quality of OCR has steadily improved ever since it was created.

\section{Template-based OCR vs AI solutions}

The most common form of template-based OCR works by the entering the coordinates of the text they want to record from a physical document. After entering this into the OCR platform the selected text is found and then recorded in a digital format like a PDF. The moment the user has finished configuring their OCR settings they have an automated solution for creating digital copies of physical documents.

\section{The next generation: AI meets OCR}

OCR tools are undergoing a quiet revolution as ambitious software providers combine them with AI. Consequently, data capturing software is simultaneously capturing information and comprehending the content. In practice this means that AI tools can check for mistakes independent of a human-user providing streamlined fault management.

Without the help of AI, such reports would need to be managed by individual employees and checked by a translator.

\section{OCR and AI: Th force for digital transformation}

Combining AI and OCR together is proving to be a winning strategy for both data capture and management. While AI-based OCR tools may not be as glamorous as other transformative technologies they will inevitably have a substantial impact on the bottom line of companies that embrace them. Reducing administrative is key to making employees more productive.

- Graphic recognition: While human and animal brains perceive objects easily, computers experience issues with the undertaking. Programming for picture acknowledgment requires profound AI. Image recognition calculations can work by utilization of similar 3D models, appearances from changed points utilizing edge discovery or by parts. Image recognition calculations are frequently prepared on huge number of pre-marked pictures with directed machine learning. Privacy concerns over image recognition and comparative innovations are controversial as these organizations can pull a huge volume of information from client photographs transferred to their online media stages. 


\section{- Image Classification using CNN:}

CNNs use relatively little pre-processing compared to other image classification algorithms. This means that the network learns the filters that in traditional algorithms were hand-engineered. This freedom from earlier information and human exertion in include configuration is a significant benefit.

When programming a CNN, the input is a tensor with shape (number of images) $\mathrm{x}$ (image height) $\mathrm{x}$ (image width) $\mathrm{x}$ (image depth). Then after passing through a convolutional layer, the image becomes abstracted toa feature map, with shape (number of images) $\mathrm{x}$ (feature map height) $\mathrm{x}$ (feature map width) $\mathrm{x}$ (feature mapchannels). A convolutional layer within a neural network should have the following attributes:

1. Convolutional kernels defined by a width and height (hyper-parameters).

2. The number of input channels and output channels (hyper-parameter).

3. The depth of the Convolution filter (the input channels) must be equal to the number channels (depth)of the input feature map.

Convolutional layers convolve the information and pass its result to the following layer. This is like the reaction of a neuron in the visual cortex to a particular improvement. Each convolutional neuron processes information just for its responsive field. Although, fully connected feedforward neural networks can be used to learn features as well as classify data, it is not practical to apply this architecture to images. A veryhigh number of neurons would be necessary, even in a shallow (opposite of deep) architecture, due to the very large input sizes associated with images, where each pixel is a relevant variable. For instance, a fully connected layer for a (small) image of size $100 \times 100$ has 10,000 weights for each neuron in the second layer. The convolution operation brings a solution to this problem as it reduces the number of free parameters, allowing the network to be deeper with fewer parameters. For instance, regardless of image size, tiling regions of size $5 \times 5$, each with the same shared weights, requires only 25 learnable parameters. By utilizing regularized loads over less parameters, the vanishing gradient and exploding gradient problems seen during backpropagation in traditional neural networks are kept away from.

\section{- You Only Look Once:}

YOLO (You Only Look Once) is a real-time object detection algorithm, which is one of the most effective object detection algorithms that also encompasses many of the most innovative ideas coming out of the computer vision research community. The issue of object identification is more perplexing than classification, which also can recognize objects but doesn't indicate where the object is located in the image. Moreover, classification doesn't work on images containing more than one object. YOLO uses a totally different approach. The algorithm applies a single neural network to the full image, and then divides the image into regions and predicts bounding boxes and probabilities for each region. These bounding boxes are weighted by the predicted probabilities. YOLO is well known because it accomplishes high precision while likewise having the option to run progressively. The algorithm "only looks once" at the image in the sense that it requires only one forward propagation pass through the neural network to make predictions. With YOLO a single CNN simultaneously predicts multiple bounding boxes and class probabilities for those boxes. YOLO trains on full images and directly optimizes detection performance. This model has variousadvantages over other object detection methods:

- YOLO is extremely fast

- YOLO sees the entire image during training and test time so it implicitly encodes contextual informationabout classes as well as their appearance. 
- YOLO learns generalizable representations of objects so that when trained on natural images and testedon artwork, the algorithm outperforms other top detection methods.

\section{Comparison Table}

\begin{tabular}{|c|c|c|c|}
\hline Solution & Objective & Mechanism & Problem Targeted \\
\hline Optical Identification & $\begin{array}{l}\text { To identify the name of } \\
\text { the drugs using AI and } \\
\text { OCR }\end{array}$ & Neural Network based & $\begin{array}{l}\text { Misreading of drugsby } \\
\text { pharmacists. }\end{array}$ \\
\hline Graphic Recognition & $\begin{array}{l}\text { To identify name of } \\
\text { drugs using Image } \\
\text { recognition }\end{array}$ & Static & $\begin{array}{l}\text { Misreading of drugsby } \\
\text { pharmacists. }\end{array}$ \\
\hline $\begin{array}{l}\text { Image classification } \\
\text { using CNN }\end{array}$ & $\begin{array}{l}\text { Use CNN and image } \\
\text { classifiers for drug } \\
\text { detection. }\end{array}$ & Machinelearningbased & $\begin{array}{l}\text { Reduce load on } \\
\text { pharmacists } \\
\text { catering to more } \\
\text { patients at once, also } \\
\text { minimising errors. }\end{array}$ \\
\hline YOLO & $\begin{array}{l}\text { Use the YOLO } \\
\text { algorithm for medicine } \\
\text { detection. }\end{array}$ & Neural Network based & $\begin{array}{l}\text { Preventing the Look- } \\
\text { Alike, Sound-Alike } \\
\text { (LASA) error. }\end{array}$ \\
\hline
\end{tabular}

\section{Conclusion}

Many people get diagnosed the wrong drugs, and this is not entirely the pharmacist's fault. Managing somany customers at once can be a tedious task and mistakes will occur. This is where the application of AI in medicine will take place, along with many other instances where AI is already being used or is planned to be used. There are many solutions and ideas which have come up to minimise and prevent such errors like for example, the 'Look-Alike, Sound-Alike' error, which is one of the most common drug diagnosis problem in the world. Algorithms like You Only Look Once (YOLO) and the efficient use of convolution neural network and image recognition will cater to many such solutions which can be finally implemented and distributed all over the world to minimise the error of misreading of drug prescriptions. 


\section{$6 \quad$ References}

1. Larios Delgado, N., Usuyama, N., Hall, A.K. et al. Fast and accurate medication identification. npj Digit.Med. 2, 10 (2019).

2. Benchmark Analysis of Representative Deep Neural Network https://arxiv.org/pdf/1810.00736.pdf

3. Constructing Azure Virtual Machine

https://intellipaat.com/blog/tutorial/microsoft-azure-tutorial/constructing-azure-virtual-machine/

4. Convolutional Neural network https://en.wikipedia.org/wiki/Convolutional_neural_network

5. H. Huang and P. Ku, "Intelligent technology enhances the friendliness of the pharmacy care service : Identification in drug prescription," 2019 International Conference on Technologies and Applications of Artificial Intelligence (TAAI), Kaohsiung, Taiwan, 2019.

6. Understanding Image recognition and its uses https://www.einfochips.com/blog/understanding-image-recognitionand-its-uses/

7. You-Only-Look-Once https://towardsdatascience.com/yolo-you-only-look-once-17f9280a47b0

8. Ting, H., Chung, S., Chen, C. et al. A drug identification model developed using deep learningtechnologies: experience of a medical center in Taiwan. BMC Health Serv Res 20, 312 (2020) 